THE ARTS

CHILD POLICY

CIVIL JUSTICE

EDUCATION

ENERGY AND ENVIRONMENT

HEALTH AND HEALTH CARE

INTERNATIONAL AFFAIRS

NATIONAL SECURITY

POPULATION AND AGING

PUBLIC SAFETY

SCIENCE AND TECHNOLOGY

SUBSTANCE ABUSE

TERRORISM AND HOMELAND SECURITY

TRANSPORTATION AND INFRASTRUCTURE

WORKFORCE AND WORKPLACE
This PDF document was made available from www.rand.org as a public service of the RAND Corporation.

Jump down to document $\nabla$

The RAND Corporation is a nonprofit research organization providing objective analysis and effective solutions that address the challenges facing the public and private sectors around the world.

\section{Support RAND}

$\underline{\text { Browse Books \& Publications }}$

Make a charitable contribution

\section{For More Information}

Visit RAND at www.rand.org

Explore RAND Testimony

View document details
Limited Electronic Distribution Rights

This document and trademark(s) contained herein are protected by law as indicated in a notice appearing later in this work. This electronic representation of RAND intellectual property is provided for non-commercial use only. Unauthorized posting of RAND PDFs to a non-RAND Web site is prohibited. RAND PDFs are protected under copyright law. Permission is required from RAND to reproduce, or reuse in another form, any of our research documents for commercial use. For information on reprint and linking permissions, please see RAND Permissions. 


\title{
The Potential Benefits and Costs of Increased Adoption of Health Information Technology
}

\author{
RICHARD HILLESTAD
}

CT-312

July 2008

Testimony presented before the Senate Finance Committee on July 17, 2008

This product is part of the RAND

Corporation testimony series.

RAND testimonies record testimony presented by RAND associates to federal, state, or local legislative committees; government-appointed commissions and panels; and private review and oversight bodies. The RAND Corporation is a nonprofit research organization providing objective analysis and effective solutions that address the challenges facing the public and private sectors around the world. RAND's publications do not necessarily reflect the opinions of its research clients and sponsors. RAND ${ }^{\circledR}$ is a registered trademark. 
Published 2008 by the RAND Corporation 1776 Main Street, P.O. Box 2138, Santa Monica, CA 90407-2138

1200 South Hayes Street, Arlington, VA 22202-5050 4570 Fifth Avenue, Suite 600, Pittsburgh, PA 15213-2665

RAND URL: http:/ / www.rand.org

To order RAND documents or to obtain additional information, contact

Distribution Services: Telephone: (310) 451-7002;

Fax: (310) 451-6915; Email: order@rand.org 


\section{Richard Hillestad, Ph.D. ${ }^{1}$ \\ The RAND Corporation}

\section{The Potential Benefits and Costs of Increased Adoption of Health Information Technology ${ }^{2}$ \\ Before the Committee on Finance \\ United States Senate}

July 17, 2008

Chairman Baucus, Ranking Member Grassley, and members of the Committee on Finance, I am honored to have the opportunity to testify before you today about the potential benefits and costs of broader adoption and effective use of Health Information Technology (or HIT).

In this testimony, I will use HIT to include an electronic medical record that replaces the paper medical record and includes such associated functions as clinical decision support for facilitating evidence-based medicine, patient tracking and reminders for preventative services, computerized physician order entry to facilitate prescribing and other physician orders, and electronic connectivity of providers (and, in some cases, among providers and patients).

I applaud the committee for including HIT as a component of getting the right care at the right time. Better health care information at the point of care, evidence-based decision support, and continuity of care across providers in time and location are some of the important promises of HIT. IT has transformed much of what we do in other domains - travel, banking, and telecommunications, for example-but despite the fact that health care is one of the largest information-intensive enterprises we know, HIT adoption in the United States is low. The health records for most people are still stored on paper, and the provider-to-provider transfer of information about a patient is still most often done by phone or fax. The questions are, then, what are the potential benefits and costs of increasing the adoption of HIT and if such adoption is worth it on balance, what actions might improve the progress of adoption?

My testimony today covers three main areas: (1) how HIT might transform care and reduce costs; (2) our estimates of the potential savings and costs if HIT is widely adopted and done right; and (3) some of the important disincentives to achieving the benefits along with possible government actions to reduce such barriers. First, what is the possible role of HIT in improving health care?

\footnotetext{
${ }^{1}$ The opinions and conclusions expressed in this testimony are the author's alone and should not be interpreted as representing those of RAND or any of the sponsors of its research. This product is part of the RAND Corporation testimony series. RAND testimonies record testimony presented by RAND associates to federal, state, or local legislative committees; government-appointed commissions and panels; and private review and oversight bodies. The RAND Corporation is a nonprofit research organization providing objective analysis and effective solutions that address the challenges facing the public and private sectors around the world. RAND's publications do not necessarily reflect the opinions of its research clients and sponsors.

${ }^{2}$ This testimony is available for free download at http://www.rand.org/pubs/testimonies/CT312/.
} 


\section{The Hope for Information Technology in Health Care-Efficient, Effective, and Consistent Health Care}

The hope of many is that the broad adoption of HIT systems with the aforementioned functionality in the United States will transform health care in terms of making it more efficient and effective simultaneously. Efficiency would be enhanced by reduced test duplication, improved drug utilization, better scheduling, reduced paper record handling, and improved claims processing and billing. Effectiveness would be enhanced by reduced errors (reduced handwriting-based errors, for example), reminders to improve preventative care, decision support for better evidence-based practice, improved management of chronic illness, and improved continuity of care for those patients seeking care away from their primary provider (such as was needed to support the mass evacuation that occurred after Hurricane Katrina). Effectiveness would also be enhanced by the quality of care assessment such systems would make possible and by improvements in the evidence base for best practices derived from the analysis of large electronic medical record databases.

But we are a long way from the broad adoption of HIT. Our own estimates of the adoption of HIT systems in the United States in 2005 indicated that about 20-25 percent of hospitals and 10-15 percent of physician offices had adopted systems that could perform at least some of the functions I just described. More recent estimates indicate that hospital-based adoption has improved but that the functionality of many of the adopted systems remains limited and that there has been relatively little change in physician adoption of HIT. Most important, only about 4 percent of physicians have HIT systems with some of the supporting functions mentioned earlier.

\section{The Potential Benefits and Costs of Broad Adoption of HIT in the United States}

The RAND study of $\mathrm{HIT}^{3}$ (begun in 2003 and published in 2005) estimated the potential benefits and costs of a much broader adoption and effective use of HIT systems. To deal with the limited evidence of savings and health benefits at that time, we developed models to extrapolate this evidence assuming 90 percent adoption, interoperability across providers, and related health care process changes, such as restructured hospital and physician office workflow, increased

\footnotetext{
${ }^{3}$ Richard Hillestad, J. Bigelow, A. Bower, F. Girosi, Robin Meili, Richard Scoville, and Roger Taylor, "Can Electronic Medical Record Systems Transform Health Care? Potential Health Benefits, Savings, and Costs," Health Affairs, Volume 24, Number 5, September/October 2005; Anthony Bower, The Diffusion and Value of Healthcare Information Technology, MG-272-1-HLTH, Santa Monica, CA: RAND, 2004; James H, Bigelow et al., Analysis of Healthcare Interventions That Change Patient Trajectories, MG-408-HLTH, Santa Monica, CA: RAND, 2005; Katya Fonkych and Roger Taylor, The State and Pattern of Health Information Technology Adoption, MG-409-HLTH, Santa Monica, CA: RAND, 2005; Federico Girosi, Robin Meili, Richard Scoville, Extrapolating Evidence of Health Information Technology Savings and Costs, MG-410-HLTH, Santa Monica, CA: RAND, 2005.
} 
preventative interventions, and aggressive team care for chronic disease. Our estimates were not best-case estimates because we used a range of evidence for each effect. However, they were also not worst-case estimates either because we did not include some negative effects. With respect to these negative effects, some papers reported some increased errors as a result of the introduction of computerized physician order entry. We assumed that these errors would decrease either because of improvements in the systems or because the systems that induced such errors would not proliferate.

Please bear in mind that our projections show the potential benefits and costs at a 90 percent adoption level and that this level might not be reached for 10-15 years based on the historical adoption rate of complex technology and the current adoption pattern of HIT. Also, the potential savings we calculate are spread among stakeholders-insurers or payers, providers, and individuals—so such savings are not necessarily savings the government might realize from programs to enhance the adoption of HIT.

Using our models, we estimated that the efficiency savings enabled by HIT could reach approximately $\$ 80$ billion per year at the 90 percent adoption level for hospitals and physicians. In turn, we estimated the costs to achieve those efficiency savings in 15 years would average about $\$ 8$ billion per year or $\$ 120$ billion total. These costs include software licenses, hardware and its maintenance, planning, training and implementation, and the reduced revenue or increased provider costs during implementation. During the 15-year adoption period, the cumulative net savings would be about $\$ 510$ billion or approximately $\$ 34$ billion per year. To put these potential savings in perspective, $\$ 80$ billion is 4 percent of the annual $\$ 2$ trillion cost of health care in the United States.

About 75 percent of the potential efficiency savings are associated with hospitals and 25 percent are associated with physician offices. In hospitals, the major savings would come from improved scheduling and shorter patient stays, reduced nursing administrative time, savings in chart administration, and better laboratory and radiology utilization (reduction in duplicate tests, for example). In physician office settings, the savings would be in drug utilization, chart administration, more efficient patient scheduling, and better laboratory and radiology utilization. We did not estimate possible savings from billing and claims administration, but we would expect those to be substantial. Furthermore, we also did not estimate possible process improvements that might further improve quality and reduce costs and that have been typical consequences of IT introduction in other industries. (Just-in-Time inventory management and Toyota's quality improvement processes are examples.) Thus, while our estimates do indicate significant potential efficiency savings, we do not consider our estimates to be overly optimistic. 
Turning to effectiveness, potential safety benefits include avoiding as many as 2.2 million adverse drug events per year and their associated costs of $\$ 4$ billion per year. The improved safety derives from reduced errors from handwriting, better allergy warnings and warnings of drug-drug interaction because of the completeness and accessibility of the electronic medical record, and better dosage monitoring. Because most prescribing occurs in physician offices, the magnitude of this benefit depends heavily on physician adoption of HIT.

About 75 percent of the national health expenditure is on chronically ill patients. HIT should enable substantial improvements in managing chronic illness. What is needed in this case is better coordination and communication across providers dealing with the multiple health impacts of a chronic illness. It also involves substantial patient screening, monitoring, and involvement. Linked HIT provides a way to coordinate support, monitor patients, and involve the patient in an effective team of care. We simulated such improved chronic illness management for four chronic illnesses ${ }^{4}$ and found the benefits from life style change, prevention, and better management of chronic illness could be 20 million fewer inpatient days, 5 million fewer emergency department visits, 9 million fewer office visits, and 20 million added workdays per year. We did not estimate cost savings from such healthier patients because it is not always true that reducing the incidence of a disease reduces health care costs-in fact, costs can go up or down depending on the effect on longevity and the occurrence of other diseases.

We estimated the costs to move hospitals and physician offices from their levels of adoption in 2005 to 90 percent adoption in 15 years to be about $\$ 120$ billion- $\$ 100$ billion for hospitals and $\$ 20$ billion for physician offices. These costs were estimated using the actual real costs of systems being implemented for physicians and hospitals and include the costs of planning and implementation, practice disruption, software, maintenance, and training.

\section{Barriers to HIT Adoption and the Possible Role of Government}

Given the potential efficiency and effectiveness savings and benefits relative to costs, it may seem surprising that the health care system has been so slow to adopt HIT. However, there are a number of significant disincentives to adoption, the primary one being what can be called a "market failure": Those who must purchase the systems (the providers-hospitals and physicians) are not the same ones receiving most of the savings (the payers) or the health benefits (the patients). Our simulation of HIT-based chronic disease management is a good example. Better management led to fewer acute episodes of the illness, which, in turn, produced significantly fewer physician office and hospital emergency department visits. Doing this reduced payer costs and produced a

\footnotetext{
${ }^{4}$ Diabetes, asthma, congestive heart failure, and chronic obstructive pulmonary disease.
} 
healthier patient, but it also reduced physician and hospital revenue for those patients and diseases. Looking at the other savings we estimated for HIT_reduced duplication of tests, reduced length of hospital stay, better drug utilization, etc.-we can see that most of the benefits are likely to go to the payers and that there is little financial incentive for the physicians and hospitals to pay the costs and undergo the disruption of HIT implementation. This market failure is a key indicator of the need for government intervention.

There is a second important issue about adoption. Current evidence is that the larger, betterendowed hospitals and physician practices are adopting HIT at a significantly higher rate than smaller, lesser-endowed hospitals and physician practices. The effect of this uneven adoption in the rate of adoption could be to increase disparities in health care. Those patients served by small offices or hospitals in rural areas or those patients seeking health care in disadvantaged urban regions might be less likely to receive the benefits of HIT-enabled health care, thus leading potentially to increases in regional health disparities as a result of this uneven adoption.

Overcoming these disincentives to adoption may require subsidies. This does not necessarily imply the outright purchase of the HIT systems for providers, but it could involve a pay-for-use incentive that compensates the physician or hospital on a per-encounter basis for the use of the HIT system. We modeled the effects of a time-limited subsidy for physicians-a per-encounter payment of $\$ 1.50$ for three years-and found that under reasonable assumptions about price elasticity, if such a subsidy were done early enough, it could increase the adoption and dramatically leverage the subsidy dollar. In this case, the cost of the subsidy was about $\$ 2$ billion over the three years and the value of the incentive over 15 years (based on the potential HIT savings previously described) would be about $\$ 16$ billion.

There may be other ways to incentivize the adoption of HIT. The institution of a pay-forperformance process that requires an electronic medical record system to measure and report the provider performance on quality of care measures-such as the appropriate ordering of screening services or the ordering of needed treatments-would provide an indirect incentive and would also place more attention on the quality of care delivered. However, this approach may also require more advanced HIT systems to be adopted to capture all the appropriate contextual information to judge quality of care.

The payers who, as I have described earlier, reap most of the projected savings of the HIT systems might be the most appropriate parties to provide subsidies. Medicare, being the largest payer, could provide significant leadership for such actions. Subsidies might also be targeted to smaller offices and hospitals and to those serving disadvantaged populations to avoid exacerbating disparities in care because of uneven adoption of HIT. 
The last issue l'd like to discuss is the difficulty of connecting the provider-based HIT systems into a network of interoperable systems supporting a patient's care wherever and whenever it is needed. Although the technology exists to do this and the national coordinator of HIT in the Department of Health and Human Services has taken important steps toward developing standards and making systems interoperable, there remain important barriers to achieving the desired interoperability. First, there is a market disincentive. The infrastructure to support interconnection includes managing master patient identification lists across providers, managing interfaces between systems, and administratively managing the network. Ideally, the funding for this would come from the users, but except for large health care systems that are under single ownership, there is little market incentive for users to share patient data with others in the network or to fund the network administration. Some early attempts to create interconnected health care networks, such as the Santa Barbara Care Data Exchange, have not succeeded once the original seed money from foundations or the government has been expended. We know there are demonstration experiments going on to understand possible financial models, but this is an area in which government action or subsidy may be needed.

Second, privacy is another concern and barrier to interconnection of HIT. This is a subject much bigger than what we can accommodate in this testimony, but we note that concern about the protection of the privacy of personal health care information is also a significant barrier to the seamless flow of patient information across the health care system. Current privacy and security protections appear to be inadequate for a national health information network and must be enhanced as the health care system becomes digitized and interconnected. An important government role will be to establish the privacy protocols that permit the networking of health information.

In closing, I have described in this testimony some of the important potential benefits of HIT in the current health care system and discussed some of the important barriers to adoption and interconnection of HIT systems. However, it is important to note that the broad adoption of HIT systems and connectivity should be considered necessary but not sufficient steps toward real health care transformation that delivers efficient and effective care at the right time. For example, the adoption of HIT systems in conjunction with comparative performance reporting could enable the development of value-based competition and quality improvement to drive transformation. We do not know what changes should or will take place after widespread HIT adoption, but it seems clear that a lengthy and uneven adoption of HIT systems that do not interoperate is likely to delay the opportunity to move closer to a transformed health care system. The government and other stakeholders have an important stake in not letting this happen. Thank you. 